\title{
Vaccine Misconceptions and Low HPV Vaccination Take-up Rates in Singapore
}

\author{
SK Tay*, KC Tesalona, N Mohamed Rashid, EYS Tai, S Mohd Najib
}

\begin{abstract}
Background: HPV vaccination in Singapore is voluntary and physician prescription-based. This study investigated the current status and intention for HPV vaccination among Singapore nurses. Materials and Methods: All female nurses in a general hospital were given an anonymous questionnaire on HPV vaccination experience and intention of vaccinating their daughters. The influence of age, knowledge and perceived-risk of cervical cancer, and cultural background on mother's intention of vaccinating their daughters was analyzed. Results: Of 2,000 nurses, $1,622(81.1 \%)$ responded and analysis was performed on 1,611 with valid data. They showed good awareness on association of cervical cancer with multiple sexual partners $(81.9 \%)$, history of

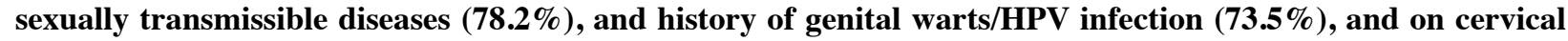
cancer preventive effects of HPV vaccination $(\mathbf{5 4 . 6 \% )})$. The prevailing misconceptions of the vaccines were: investigational nature (38.9\%), side effects $(\mathbf{2 7 . 9 \%})$ and indicated for women at high risk for cervical cancer (20.5\%). Conclusions: Misconceptions on the nature, role and safety of HPV vaccines low vaccine up-take rates and daughters. Dissemination of adequate and accurate HPV vaccine information and a review for school-based vaccination are needed for optimal delivery of HPV vaccines in Singapore.
\end{abstract}

Keywords: Cancer prevention - cervical cancer - culture - knowledge - school-based mass vaccination - vaccine safety

Asian Pac J Cancer Prev, 16 (12), 5119-5124

\section{Introduction}

Cervical cancer is the third most common cancer among women worldwide, with $85 \%$ of the burden in developing countries (Ferlay et al., 2010). The incidence and mortality rates of cervical cancer have been declining in countries where women participate in comprehensive screening. In Singapore, the national cervical screening program using cervical cytology test was introduced in 2004. Cervical cancer now ranks the $10^{\text {th }}$ among the top 10 commonest cancer in women in Singapore. The most recent estimate of aged standardized incidence rate at 7/100,000 women is comparable to Australia (Canfell et al., 2006; National Register of Diseases Office, 2014). The efficacy of cytology screening program for cervical cancer prevention is closely related to the proportion of eligible women participating the screening (Sasieni et al., 2003). After decades of cervical screening in Singapore, only $47.9 \%$ of eligible women participated in regular cytology screening (Ministry of Health Singapore, 2014). Additional measures are needed to optimize the effort of controlling burden of cervical cancer in Singapore.

The etiology of cervical cancer is related to human papillomavirus (HPV) infection. There are $15 \mathrm{HPV}$ subtypes with oncogenic property. Of these, HP-16 and HPV-18 are responsible for more than $70 \%$ of all cervical cancer (Bosch et al., 2002; de Sanjose et al., 2010). Prophylactic vaccines against HPV-16 and HPV-18 infection are highly efficacious and have been available for mass vaccination since 2006 (The Future II Study Group, 2007; Paavonen et al., 2009; Schiller et al., 2012). Some countries have implemented national school-based vaccination for adolescent girls between 12 and 14 years old. The vaccine uptake rates for these programs exceed $70 \%$ (Garland et al., 2011). Other communities, including Singapore, have adopted an individualized vaccination approach. Ministry of Health, Singapore recommends HPV vaccination for girls and women between the age of 9 and 26 years old and the cost of vaccination is payable through Medisave account. Being an individual initiated vaccination scheme, participation of vaccination is voluntary and not notifiable for official registration. Data on the up-take rate of HPV vaccination among eligible girls and young women in Singapore is therefore nonexisting and has been estimated to be low.

Many factors have been implicated in low screening and HPV vaccine uptake rates. Apart from accessibility to screening and vaccination, socio-economic status, cultural factors and knowledge about HPV and cervical cancer of women seem to influence women's decision to participate in these programs (Cates et al., 2009; Hughes et al., 2009; Gamble et al., 2010; Jaspers et al., 2011; Lechuga et al., 
2011; Marlow, 2011). The impact of these factors on HPV vaccination up-take rate in Singapore, a multiracial and multicultural society of relative affluence is unknown. We were particularly interested in the potential significance of knowledge of HPV, cervical cancer and cultural factors on acceptance of HPV vaccination. In this study of nurses in a tertiary hospital, a population of similar socio-economic status, we investigated the up-take rate of HPV vaccination and reasons for declining vaccination for themselves and their daughters.

\section{Materials and Methods}

This study was approved by Singhealth Institutional Review Board for clinical research. Participation in the study was absolutely voluntary.

A printed questionnaire was delivered to all the female nurses in 24 wards in a large tertiary general hospital in Singapore. The questionnaire, without personal identifiers, asked for the following information: age category and years in nursing practice, ethnic origin and religion, statement on the factor most important in identifying cultural value, status on marital and sexual relationship, 10 true-false statements on common knowledge about epidemiology and risk factors on cervical cancer, and their history, intention for and reasons against HPV vaccination. Subjects were given three days to answer the questionnaire in private. Subjects returned the completed questionnaire form by dropping it into a sealed box in each ward. Information on the questionnaire was transcribed into a spread sheet for data analysis.

\section{Statistical analysis}

IBM SPSS version 22 was used for data analysis. Descriptive analysis on frequency distribution of demographic characteristics of the responders was performed. One point was assigned to each correct answer on knowledge of cervical cancer and an aggregated score was computed for each responder. The minimum aggregated score was 0 and the maximum score was 10 . Chi-square analysis was performed for test of significance of difference between attributes including knowledge score and cultural groupings, between behavior and decision on HPV vaccination.

Cultural grouping was defined by combination of ethnicity and religious faith.

\section{Results}

A total of 2000 questionnaires were sent and 1622 $(81.1 \%)$ nurses responded. Of these, 1296 (79.9\%) were ordinary Singapore residents, $315(19.4 \%)$ were guest workers and $11(0.7 \%)$ did not state their nationality status. The median score on knowledge in epidemiology of cervical cancer was 5 (range 0-9) out of a maximum score of 10 . There was no difference in distribution of score between age groups $(\mathrm{p}=0.527)$ or ethnic groups $(\mathrm{p}=0.362)$. Overall, the responders were aware that a woman's risk of cervical cancer was associated with multiple sexual partners $(1329 / 1611$ or $81.9 \%)$, history of sexually transmissible diseases (1269/1611 or $78.2 \%)$ and history of genital warts or HPV infection (1192/1611 or $73.5 \%)$. However, on the self-perceived risk for cervical cancer, $6 \%(97 / 1622)$ reported high risk, $41.6 \%$ $(675 / 1622)$ reported low risk and 50.6\% (821/1662) were unsure of their risk. Similar pattern of risk perception was seen among nurses 25 years old or younger.

Of 1622 responders, $1233(76.0 \%)$ stated that specific cultural and traditional practices in their family were important to them. The most important factors identifying the cultural value, in descending order of prevalence among responders, were religion (826 $(50.9 \%))$, nationality $(384(23.7 \%))$ and ethnicity (342 $(21.1 \%))$. For further analysis on influence of culture in behavior of participating HPV vaccination, responders were categorized into ethnic-religion groupings.

There were 1611 responders provided valid information on HPV vaccines and formed the subjects for this analysis. There was a high prevalence of misconception and uncertainty on HPV vaccines among responders. Eight hundred and eighty responders $(54.6 \%)$ were aware that HPV vaccination could reduce women's risk of cervical cancer, but 627 (38.9\%) thought that the vaccines were investigational drugs and another $450(27.9 \%)$ believed that serious side effects of the vaccines were common. Five hundred and twenty $(32.2 \%)$ responders reported that HPV vaccines were indicated for young women only and $330(20.5 \%)$ believed that the vaccines were meant for women at high risk for cervical cancer. Only 163 $(10.1 \%)$ responders reported that HPV vaccine was also indicated for boys.

Overall, 142 (8.8\% or $142 / 1611$ cases) had received HPV vaccination, $202(12.5 \%)$ planned to receive it within the next 12 months, $721(44.5 \%)$ were undecided and $557(34.3 \%)$ declined the vaccination. Figure (1) showed the most commonly cited reasons for declining vaccination: inadequate information (49.4\%), unproven vaccine effectiveness $(23.5 \%)$ and wrong age $(25.5 \%)$.

Table 1.Perceived Risk for Cervical Cancer: Agreement between Mother's Personal and Daughter's Risk

\begin{tabular}{lcrrrr}
\hline My own risk & \multicolumn{5}{c}{ My daughter's risk } \\
& Not stated & High & Low & Unsure & Total \\
\hline High & 1 & 41 & 11 & 44 & 97 \\
Low & 9 & 8 & 433 & 230 & 680 \\
Unsure & 6 & 7 & 29 & 784 & 826 \\
Not available & 19 & 0 & 0 & 0 & 19 \\
Total & 35 & 56 & 473 & 1058 & 1622 \\
\hline
\end{tabular}

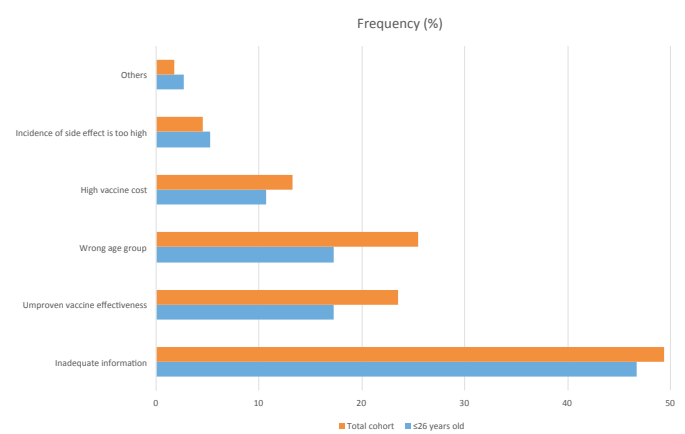

Figure 1. Frequency Distribution of Reasons on Declinig HPV Vaccination 
Vaccine Misconceptions and Low HPV Vaccination Take-up Rates in Singapore

Table 2. Distribution of Characteristics of 247 Mothers with Daughters between 10 and 26 Years Old

\begin{tabular}{|c|c|c|c|c|c|c|c|}
\hline \multirow{2}{*}{\multicolumn{2}{|c|}{$\begin{array}{l}\text { Responder's } \\
\text { characteristics }\end{array}$}} & \multirow[t]{2}{*}{ No. of cases } & \multicolumn{5}{|c|}{ Intention on daughter's vaccination } \\
\hline & & & Had been & To be vaccinated & Undecided & To vaccinate & Against \\
\hline \multirow[t]{7}{*}{ Age } & $25-29$ & 19 & 0 & 3 & 2 & 5 & 9 \\
\hline & $30-34$ & 24 & 1 & 0 & 4 & 8 & 11 \\
\hline & $35-39$ & 25 & 1 & 0 & 9 & 4 & 11 \\
\hline & $40-44$ & 40 & 0 & 2 & 11 & 8 & 19 \\
\hline & $45-49$ & 49 & 2 & 7 & 10 & 7 & 23 \\
\hline & $50-54$ & 50 & 5 & 6 & 11 & 4 & 24 \\
\hline & $>54$ & 40 & 2 & 2 & 9 & 3 & 24 \\
\hline \multicolumn{7}{|c|}{$P$ value } & 0.147 \\
\hline \multicolumn{8}{|c|}{ Nursing duration (years) } \\
\hline & $<5$ & 8 & 0 & 0 & 0 & 1 & 7 \\
\hline & 5-Sep & 32 & 1 & 3 & 4 & 7 & 17 \\
\hline & Oct-14 & 38 & 1 & 1 & 10 & 11 & 15 \\
\hline & $15-19$ & 24 & 1 & 0 & 7 & 5 & 11 \\
\hline & $20-24$ & 52 & 1 & 5 & 15 & 8 & 23 \\
\hline & $>24$ & 93 & 7 & 11 & 20 & 7 & 48 \\
\hline P val & & & & & & & 0.129 \\
\hline \multicolumn{8}{|c|}{ Cervical cancer knowledge score } \\
\hline & $<3$ & 44 & 0 & 6 & 6 & 9 & 23 \\
\hline & 4-Jun & 165 & 8 & 11 & 40 & 23 & 83 \\
\hline & 6-Sep & 38 & 3 & 3 & 10 & 7 & 15 \\
\hline P val & & & & & & & 0.703 \\
\hline
\end{tabular}

Table 3. Mother's Intention of Vaccinating Daughters by Cultural Grouping

\begin{tabular}{|c|c|c|c|c|c|}
\hline \multirow{2}{*}{$\begin{array}{l}\text { Responder's } \\
\text { characteristics }\end{array}$} & \multirow[t]{2}{*}{ No. of cases } & \multicolumn{4}{|c|}{ Intention on daughter's vaccination } \\
\hline & & $\begin{array}{c}\text { To be vaccinated } \\
\text { ( }<12 \text { months })\end{array}$ & Undecided & $\begin{array}{c}\text { To vaccinate } \\
\text { only in a school- } \\
\text { based program }\end{array}$ & $\begin{array}{c}\text { Against } \\
\text { vaccination }\end{array}$ \\
\hline
\end{tabular}

Cultural category

Malay Muslim

Chinese Buddhist

Chinese Christian

Chinese catholic

Chinese others

Indian Hindu

Indian Muslim

Asian catholic

Other catholic

Others

$\begin{array}{rl}60 & 1 \\ 29 & 2 \\ 29 & 5 \\ 12 & 0 \\ 32 & 0 \\ 27 & 0 \\ 7 & 0 \\ 17 & 1 \\ 11 & 0 \\ 23 & 2\end{array}$

$\begin{array}{ll}1 & 3 \\ 2 & 3 \\ 5 & 4 \\ 0 & 0 \\ 0 & 2 \\ 0 & 5 \\ 0 & 0 \\ 1 & 0 \\ 0 & 0 \\ 2 & 3\end{array}$

3
3
4
0
2
5
0
0
0
3

6
5
7
3
10
6
3
5
3
8

\begin{tabular}{rr}
14 & 36 \\
2 & 17 \\
3 & 10 \\
2 & 7 \\
3 & 17 \\
3 & 13 \\
0 & 4 \\
4 & 7 \\
3 & 5 \\
5 & 5 \\
& 0.692 \\
\hline
\end{tabular}

Among 446 responders below 25 years old, 43 $(9.6 \%)$ had received vaccination, $60(13.5 \%)$ planned to take vaccination within the next 12 months, 218 (48.9\%) remained undecided and 125 (28.0\%) declined vaccination. The most common reasons for declining vaccination were similar to the total cohort as shown in Figure (1) were: inadequate information (56\%), unproven vaccine effectiveness $(20.8 \%)$, age $(20.8 \%)$ and high vaccine cost $(12.8 \%)$.

There were 247 women who had at least one daughter in the age band of 10 to 25 years old. There was a strong agreement of the mother's assessment of her own risk and the daughter's risk of cervical cancer (Table 1). It was noteworthy that $48.2 \%(119 / 247)$ of these mothers were unsure or uncommitted on their own risk of cervical cancer and $91.6 \%$ of this group of mothers (109/119) expressed uncertainty about their daughter's risk.

Of these 247 responders, 11 (4.4\%) had brought the

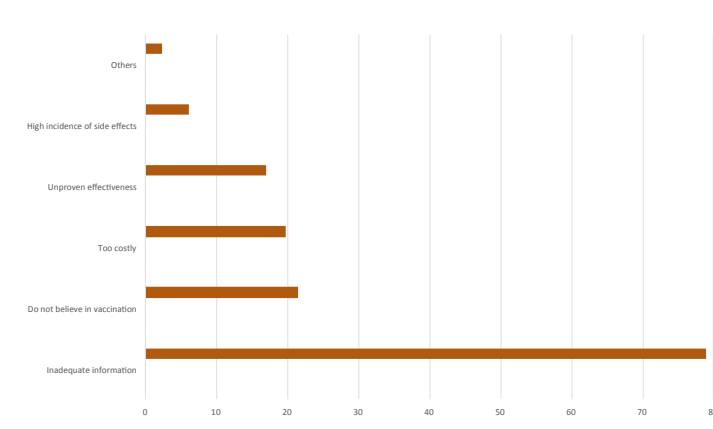

Figure 2. Reasons for Not Vaccinating Daughters

daughters for vaccination, $20(8.1 \%)$ intended to take the vaccination within the next 12 months, 56 (22.7\%) were undecided, 39 (15.8\%) would accept vaccination if it was a school-based program, and 121 (49.0\%) decided against vaccination of the daughters. These decisions were not influenced by the age of the women, the duration of 
nursing years, women's score on the knowledge of cervical cancer, or cultural group (Table 2). The most common reasons for deciding against vaccinating daughters (Figure 2) were inadequate information $(78.9 \%)$. There was no significant impact of cultural grouping on the mother's decision of vaccinating the daughters (Table 3).

\section{Discussion}

Large clinical trials have established that HPV vaccines are highly efficacious in preventing persistent infection by HPV-16 and HPV-18 and high-grade neoplasia of the cervix. These benefits were confirmed in the real world experience after introduction of mass vaccination in Australia, the United Kingdom and Denmark (Brotherton et al., 2011; Mesher et al., 2013; Baldur-Felskov et al., 2014; Kavanagh et al., 2014; Pollock et al., 2014). Concerted effort is needed to implement HPV vaccination as widely and as quickly as possible in order to maximize the benefit of the vaccines on controlling the burden of cervical cancer nationally and globally.

The up-take rate of HPV vaccination among organized school-based universal vaccination program is much higher than the physician-based vaccination program (Department of Health 2012; Laemmle-Ruff et al., 2013; Sacks et al., 2014). In Australia, vaccination up-take rate was more than $80 \%$ among $12-13$ years-old in schoolbased program compared to 7-49\% of 18-26 years-old in General Practitioner-based catch up program (Australian Health Authority 2014; Sacks et al., 2014). The uptake rate is likely to be even lower in countries with no organized vaccination program.

In our study, HPV vaccination up-take rate among nurses was $8.8 \%$ (142/1611 cases) overall and 9.6\% (43/446) for young nurses below 26 years. In a similar situation, France recommended HPV vaccination without an organized program, the up-take rate of vaccination was $6.5 \%$ for young women between 18 and 23 years old in 2010 (Ganry et al., 2013).

We were disappointed by these findings as the awareness among them was high on the association of women's risk of cervical cancer with multiple sexual partners $(1329 / 1611$ or $81.9 \%)$, history of sexually transmissible diseases $(1269 / 1611$ or $78.2 \%)$ and history of genital warts or HPV infection (1192/1611 or $73.5 \%)$, and the protective benefit of HPV vaccination $(54.6 \%$ or $880 / 1622)$. More worryingly, $34.3 \%$ of the entre cohort and $28.0 \%$ of the young nurses decisively declined HPV vaccination. We further found that only $4.4 \%$ $(11 / 247)$ of nurses had taken their young daughters for HPV vaccination and $49 \%$ had declined vaccinating the daughters, a similar finding to a 2011 survey of lay public in Singapore where only $2 \%$ of mothers had taken their daughters at the age of 9-14 years old for HPV vaccination (Koh et al., 2013). Extrapolation of findings from these two studies on the current status of HPV vaccination indicated that the great majority of young women in Singapore remained vulnerable to HPV-16 and HPV-18 infection and their associated risk of cervical neoplasia and cancer.

Literature shows that, among non-attendance for HPV vaccination, lack of knowledge on HPV and cervical cancer and low perception of vaccine protection against cervical cancer were frequently found to be significant barriers (Mortensen GL 2010; Waller 2012; Wong et al., 2013). We were therefore puzzled by our responders' behavior. Our data further showed that our responders' behavior was not influenced by the chronological age, years of experience in nursing or culture as defined by the ethnicity and religion groupings.

We, however, observed that only $6 \%$ of our responders considered themselves at high risk of cervical cancer. The remaining responders were almost equally split between low risk and uncommitted for the risk of cervical cancer. It is understandable that women who perceived themselves at low risk would not see the need for HPV vaccination but one would have thought that, since $54.6 \%$ of the responders were aware that HPV vaccination could reduce women's risk of cervical cancer, those who were unsure of their risk for cervical cancer would take the advantage of protective benefit of HPV vaccination. Our observation suggested that knowledge alone was not sufficient to drive behavioral changes. Our findings agreed with the report from USA that HPV knowledge level alone was not associated with the willingness of mothers to vaccinate their daughters against HPV (Lai et al., 2013). A recent systemic review also concluded that evidence is lacking for a significant impact of interventional education on HPV vaccination up-take rate (Fu et al., 2014).

Nonetheless, public education remains pertinent in Singapore in addressing the high reported rate of lack of vaccine information (78\%) and prevalent misconceptions that the vaccines were investigational drugs (38.9\%) and serious side effects were common $(27.9 \%)$, and that HPV vaccines were indicated for young women at risk of cervical cancer $(32.2 \%)$. These beliefs were unfounded. The two commercially available vaccines had been licensed for clinical usage by the Singapore regulatory authority since 2006 and 2007. Both clinical trials and post-marketing pharmaco-surveillance have clearly established the vaccine safety (Slade et al., 2009; WHO 2014). We believe that these misconceptions and skepticism about the vaccines were important reasons for the low up-take rate of HPV vaccination among our responders. I has been shown that, within an organized HPV vaccination program, vaccine knowledge was an important determinant for participation of vaccination by targeted girls (Gefenaite et al., 2012).

Let alone the controversy in efficacy, the impact of education on modifying women's behavior in participating HPV vaccination is likely a slow process as witnessed by the experience of hepatitis B vaccination. Moreover, a participation rate of $50 \%$ or more of eligible women in HPV vaccination is required to demonstrate the costeffectiveness of the vaccine in reducing the incidence of cervical cancer in Singapore (Lee et al., 2011). To achieve this, the advantage and efficacy of school-based HPV vaccination cannot be over-emphasized. Indeed, in Malaysia where the ethnic-religion mix was not dissimilar to Singapore, a school-based HPV vaccination program achieved an up-take rate of $87 \%$ (Ezat et al., 2013).

Conclusion: Despite being well-educated healthcare 
workers with good knowledge in the role of HPV in cervical cancer development, the subjects of this study reported a low up-take rate of HPV vaccination and only a small proportion of them had taken their daughters for the vaccination. The widespread misconceptions about HPV vaccines included experimental preparations, indicated for women at high-risk of cervical cancer, and prevalent adverse medical conditions. Our findings, taken together with the similar results of a smaller survey in 2011 of lay public in Singapore, raised a concern that, under the current voluntary prescription-based vaccination scheme, a great number of young women in Singapore remained unprotected against HPV-16 and HPV-18 infection and their associated cervical neoplasia and cancer. These results highlighted the urgent need for efforts in providing accurate vaccine information and for public health policy makers to review the role of a school-based mass vaccination of young adolescent girls in optimizing the delivery of the benefits of HPV vaccination in Singapore.

\section{References}

Australian Health Authority (2014). Immunisation coverage, 2012. CDI 2014; 38, 226. (Access on 4 December 2014).

Baldur-Felskov B, Dehlendorff C, Munk C, Kjaer SK (2014). Early impact of human papillomavirus vaccination on cervical neoplasia-nationwide follow-up of young danish women. JNCI J Natl Cancer Inst, 106, 460.

Bosch FX, Lorincz A, Munoz N, Meijer CJ, Shah KV (2002). The causal relation between human papillomavirus and cervical cancer. J Clin Pathol, 55, 244-65.

Brotherton JM, Fridman M, May CL, et al (2011). Early effect of the HPV vaccination programme on cervical abnormalities in Victoria, Australia: an ecological study. Lancet, 377, 2085-92.

Canfell K, Sitas F, Beral V (2006). Cervical cancer in Australia and the United Kingdom: comparison of screening policy and uptake, and cancer incidence and mortality. Med J Aust, 185, 482-6.

Cates JR, Brewer NT, Fazekas KI, Mitchell CE, Smith JS (2009). Racial differences in HPV knowledge, HPV vaccine acceptability, and related beliefs among rural, Southern women. J of Rural Health, 25, 93-7.

de Sanjose S, Quint WGV, Alemany L, et al (2010). Human papillomavirus genotype attribution in invasive cervical cancer: a retrospective cross-sectional worldwide study. Lancet Oncol, 11, 1048-56.

Department of Health (2012). Annual HPV vaccine coverage in England in 2010/2011. https://www.wp.dh. gov.uk/immunisation/files/2012/03/120319_HPV_ UptakeReport2010-11-revised_acc.pdf

Ezat SWP, Hod R, Mustafa J, Dali AZHM, et al (2013). National HPV immunisation programme: knowledge and acceptance of mothers attending an obstetrics clinic at a teaching hospital, Kuala Lumpur. Asian Pac J Cancer Prev, 14, 2991-9.

Ferlay J, Shin HR, Bray F, et al (2010). Estimates of worldwide burden of cancer in 2008: GLOBOCAN 2008. Int J Cancer, 127, 2893-917.

Fu LY, Bonhomme LA, Cooper SC, Joseph JG, Zimet GD (2014). Educational interventions to increase HPV vaccination acceptance: a systematic review. Vaccine, 32, 1901-20.

Gamble HL, Klosky JL, Parra GR, Randolph ME (2010). Factors Influencing familial decision-making regarding human papillomavirus vaccination. J Pediat Psychol, 35, 704-15.
Ganry O, Bernin-Mereau AS, Gignon M, Merlin-Brochard J, Schmit JL (2013). Human papillomavirus vaccines in Picardy, France: Coverage and correlation with socioeconomic factors. Epidemiol Pub Health, 61, 447-54.

Garland S, Skinner SR, Brotherton JM (2011). Adolescent and young adult HPV vaccination in Australia: achievements and challenges. Prev Med, 53, 29-35.

Gefenaite G, Smit M, Nijman HW, et al (2012). Comparatively low attendance during Human Papillomavirus catch-up vaccination among teenage girls in the Netherlands: Insights from a behavioral survey among parents. BMC Public Health, 12, 498.

Hughes J, Cates JR, Liddon N, et al (2009). Disparities in how parents are learning about the human papillomavirus vaccine. Cancer Epidemiol Biomarkers Prev, 18, 363-72.

Jaspers L, Budiningsih S, Wolterbeek R, Henderson FC, Peters AAW (2011). Parental acceptance of human papillomavirus (HPV) vaccination in Indonesia: A cross-sectional study Vaccines, 29, 7785-93.

Kavanagh K, Pollock KGJ, Potts A, et al (2014). Introduction and sustained high coverage of the HPV bivalent vaccine leads to a reduction in prevalence of HPV 16/18 and closely related HPV types. British J Cancer, 110, 2804-11.

Koh JY, Fock CL, Thilagaratnam S (2013). Education efforts to drive uptake of HPV vaccination in Singapore. EUROGIN 4 November2013; Abstract P16-7. (Access on 4 December 2014).

Laemmle-Ruff I, Barbaro B, Brotherton J (2013). Human papillomavirus vaccine national catch-up program-insights into under-notification. Aust Fam Physician, 42, 880-4.

Lai JY, Tinker AV, Cheung WY (2013). Factors influencing the willingness of US women to vaccinate their daughters against the human papillomavirus to prevent cervical cancer. Med Oncol, 30, 582.

Lechuga J, Swain GR, Weinhardt LS (2011). The cross-cultural variation of predictors of human papillomavirus vaccination intentions. J Women's Health, 20, 225-30.

Lee VJ, Tay SK, Teoh YL, Tok MY (2011). Cost-effectiveness of different human papillomavirus vaccines in Singapore. BMC Public Health, 11, 203.

Ministry of Health Singapore (2014). Preventive health screening. http://www.moh.gov.sg/content/moh_web/ home/statistics/Health_Facts_Singapore/Preventive_ Health_Screening.html

Marlow L (2011). HPV vaccination among ethnic minorities in the UK: knowledge, acceptability and attitudes. British $J$ Cancer, 105, 486-92.

Mesher D, Soldan K, Howell-Jones R, et al (2013). Reduction in HPV 16/18 prevalence in sexually active young women following the introduction of HPV immunisation in England. Vaccine, 32, 26-32.

Mortensen GL (2010). Drivers and barriers to acceptance of human-papillomavirus vaccination among young women: a qualitative and quantitative study. BMC Public Health, 10,68 .

National Registry of Diseases Office (2014). Trends in cancer incidence in Singapore 2007-2011. Page 10.

Paavonen J, Naud P, Salmeron J, et al (2009). Efficacy of human papillomavirus (HPV)-16/18 AS04-adjuvanted vaccine against cervical infection and precancer caused by oncogenic HPV types (PATRICIA): final analysis of a double-blind, randomised study in young women. Lancet, 374, 301-14.

Pollock KG, Kavanagh K, Potts A, et al (2014). Reduction of low- and high-grade cervical abnormalities associated with high uptake of the HPV bivalent vaccine in Scotland. $B r J$ Cancer, 111, 1824-30.

Sacks RJ, Andrew J, Copas AJ, Wilkinson DM, Robinson AJ 
(2014). Uptake of the HPV vaccination programme in England: a cross-sectional survey of young women attending sexual health services. Sex Transm Infect, 90, 315-21.

Sasieni P, Adams J, Cuzick J (2003). Benefit of cervical screening at different ages: evidence from the UK audit of screening histories. Br J Cancer, 89, 88-93.

Schiller JT, Castellsague X, Garland S (2012). A review of clinical trials of human papillomavirus prophylactic vaccines. Vaccine, 30, 123-38.

Slade BA, Leidel L, Vellozzi C, et al (2009). Post-licensure safety surveillance for quadrivalent human papillomavirus recombinant vaccine. JAMA, 302, 750-7.

The Future II Study Group (2007). Effect of prophylactic human papillomavirus L1 virus-like-particle vaccine on risk of cervical intraepithelial neoplasia grade 2, grade 3, and adenocarcinoma in situ: a combined analysis of four randomised clinical trials. Lancet, $\mathbf{3 6 9}, 1861-68$.

Waller J, Jackowska M, Marlow L, et al (2012). Exploring age differences in reasons for Non attendance for cervical screening: a qualitative study. $B J O G, \mathbf{1 1 9}, 26-32$.

WHO (2014). Global advisory committee on vaccine safety statement on the continued safety of HPV vaccination. http://www.who.int/vaccine_safety/committee/topics/hpv/ GACVS_Statement_HPV_12_Mar_2014.pdf

Wong MCS, Lee A, Ngai KLK, Chor JCJ, Chan PKS (2013). Knowledge, attitude, practice and barriers on vaccination against human papillomavirus infection: a cross-sectional study among primary care physicians in Hong Kong. PLOS ONE, 8, 71827 . 